\title{
642 DECREASED HOST PKC-DELTA IS ASSOCIATED WITH THE T CELL-INFLAMED TUMOR MICROENVIRONMENT AND IMPROVES ANTI-TUMOR IMMUNITY
}

Kyle Cron*, Ayelet Sivan, Keston Aquino-Michaels, Emily Higgs, Jessica Fessler, Seoho Lee, Randy Sweis, Thomas Gajewski. University of Chicago, Chicago, IL, United States

Background Favorable clinical responses to immunotherapy have been correlated with a $\mathrm{T}$ cell-inflamed tumor microenvironment. The degree of spontaneous immune infiltration in tumors varies widely between individual patients. We hypothesized that germline polymorphisms in immune regulatory genes may affect the host immune response to solid tumors, similar to their influence on autoimmune susceptibility.

Methods Melanoma TCGA RNAseq and germline SNP data were utilized to identify germline polymorphisms associated with the magnitude of an immune gene signature score. The top GWAS hit associated with increased immune gene expression in melanoma was SNP rs1483185 ( $\mathrm{p}=8.812 \mathrm{e}-08$, Bonferroni corrected $<0.05$ ), within the PKC-delta gene. Using a lymphoblastic cell line GTEX database, this SNP was associated with lower expression PKC-delta, implying a loss of function phenotype. Germline mutations in PKC-delta had previously been associated with familial lupus. To study the role of PKC-delta in anti-tumor immunity, knockout hematopoietic cells and conditional knockout mice were utilized, and implanted tumors were monitored along with detailed immune response analysis.

Results B16.SIY tumors grew more slowly in chimeras reconstituted with PKC-delta-/- bone marrow compared to WT bone marrow, and this effect was dependent on CD8-beta+ cells. $\mathrm{T}$ cell priming in the tumor-draining lymph node was comparable in WT and KO hosts. However, tumors in PKCdelta-/- bone marrow chimeras had increased numbers of CD8 $+\mathrm{T}$ cells in the tumor at endpoint, and also responded better to anti-PD-L1 therapy. Single cell RNAseq of the tumor microenvironment revealed that PKC-delta loss primarily altered gene expression in myeloid cell subsets, leading to to increased expression of M1 associated genes and decreased expression of M2 associated genes in PKC-delta-/- chimeras. To follow up further, a conditional PKC-delta $\mathrm{KO}$ mouse was developed and crossed to the hematopoietic Vav1-iCre and also to LysM-Cre transgenic mice. In both instances, immunemediated tumor control was improved, demonstrating that loss of PKC-delta in the myeloid compartment is sufficient to recapitulate the phenotype.

Conclusions Our results demonstrate that germline variants in immune regulatory genes can profoundly affect anti-tumor immunity and the efficacy of PD-1/PD-L1 blockade. In particular, the myeloid-expressed molecule PKC-delta plays an important regulatory role such that decreased expression/activity mediates improved anti-tumor immunity by altering the M1/M2 ratio. The development of pharmacologic approaches to phenocopy this loss of function phenotype may be attractive to pursue as a novel therapeutic strategy.

Ethics Approval University of Chicago IRB Protocol 15-0837

http://dx.doi.org/10.1136/jitc-2021-SITC2021.642 